\title{
Tripanossomose em equinos na região sul do Brasil
}

\author{
Trypanosomosis in equines in southern Brazil
}

\author{
Aleksandro Schafer da Silva', Olmiro Adair Silveira de Andrade Neto ${ }^{2}$, Marcio Machado Costa ${ }^{1}$, Patrícia \\ Wolkmer ${ }^{1}$, Cinthia Melazzo Mazzantti ${ }^{3}$, Janio Morais Santurio ${ }^{3}$, Sonia Terezinha dos Anjos Lopes ${ }^{3}$ \& \\ Silvia Gonzalez Monteiro ${ }^{3}$
}

\begin{abstract}
Background: Trypanosoma evansi (T. evansi) is a protozoan which causes trypanosomosis in livestock in many countries of Southeast Asia, Africa and South America. Patterns of disease vary from acute epidemics with high case-fatality rates to subclinical and/or chronic disease in endemic animal populations. It is a problem of great economic importance due to the death of sick animals and high cost of treatment. This article aims to review the outbreaks of the infection by T. evansi in horses that occurred in southern Brazil.

Review: These outbreaks were discussed in terms of epidemiology, clinical signs, laboratory tests, pathological findings, diagnosis and treatment by addressing the differences between the cases occurred in the state of Rio Grande do Sul and in other Brazilian states. The outbreaks due to T. evansi in livestock animals are endemic in warm-climate areas. At the Rio Grande do Sul state, most of the equine trypanosomosis occurs in the summer. This can be easily explained by the high number of bloodsucking insects, which are responsible for the mechanical transmission of the flagellate among the animals. Clinical signs such as progressive weight loss, lethargy, incoordination, instability, atrophy and paralysis of the hind limbs, difficulty in standing and walking, subcutaneous edema and abortion are often reported in T. evansi-infected equines. Anemia is the clinical alteration most observed in these infections, although its pathogenicity still remains unclear. In the present study anemia was associated with lipid peroxidation and decrease in serum iron levels and in acetylcholinesterase activity. Necropsy alterations found in the outbreaks reported in the Rio Grande do Sul state are commonly described in infections by T. evansi, except by the neurological alterations as necrotizing encephalitis of the white matter associated with edema, demyelinization and perivascular lymphoplasmocytic infiltrate. The diagnosis of the equine trypanosomosis was based on morphology and biometry of the trypomastigote forms in peripheral blood smears stained with Quick Panoptic or Giemsa methods, immunohistochemic, xenodiagnostic, and PCR T. evansi-specific. A new therapeutic protocol using diminazene aceturate at a dose of $7 \mathrm{mg} \mathrm{kg}^{-1} \mathrm{was}$ tested in one of the outbreaks. This approach cured all the infected animals.

Conclusion: Although T. evansi was diagnosed for the first time in Rio Grande do Sul state in 2002, veterinary clinicians have reported clinical signs such as paralysis of the pelvic members, fever and weight loss since the 80's and 90's. Therefore, the lack of knowledge of the disease might have been responsible for the unpublished data. Another hypothesis is the suspect of babesiosis, since both illnesses have marked anemia and hyperthermia. Moreover, as the diminazene aceturate has trypanocidal and babesicidal action, animals may have been misdiagnosed with babesiosis and may have recovered from the T. evansi infection. Only few researches on trypanosomosis are found in the southern region of Brazil. Prevalence studies with more sensible techniques are necessary in order to clarify the spread of the disease and the economic losses that it causes to farmers.
\end{abstract}

Keywords: Trypanosoma evansi, equines, diagnosis, treatment.

Descritores: Trypanosoma evansi, equinos, diagnóstico, tratamento. 
I. INTRODUÇÃO

\section{SURTOS DE TRIPANOSSOMOSE NO SUL DO BRASIL}

\section{NOVO CASO: TRYPANOSOMA EVANSI EM EQUINO NO RS}

IV. DISCUSSÃO

V. CONSIDERAÇÕES FINAIS

\section{INTRODUÇÃO}

Trypanosoma evansi é um protozoário da seção salivaria, agente etiológico da doença conhecida como Mal das Cadeiras ou Surra em equinos [36]. Apresenta ampla distribuição geográfica parasitando equinos, camelos, bovinos, caprinos, ovinos, suí-nos, cães, gatos, búfalos, elefantes, capivaras, quatis, antas, veados, pequenos roedores silvestres [36] e humanos [19]. T. evansi pode ser transmitido mecanicamente por insetos e morcegos hematófagos [21].

Em cães e equinos, o protozoário produz sinais clínicos tais como anemia, edema de pernas e partes baixas, letargia, perda de apetite, emagrecimento, febre intermitente, lacrimejamento e aborto [5,23,28,33-35,46]. Para o diagnóstico da tripanossomose podem ser utilizados exames parasitológicos, imunológicos e moleculares [38,43,44]. No Brasil, quando constatada infecção por T. evansi, o aceturato de diminazeno é o produto comumente utilizado no tratamento de animais domésticos, por apresentar altos índices terapêuticos, apesar do grande número de casos com recidiva da doença após terapia $[4,27]$.

No Brasil, o T. evansi afeta principalmente equinos e a prevalência da infecção varia de região para região $[14,20]$. A doença é enzoótica em equinos do Pantanal matogrossense [33]. Já na região sul do Brasil, foram diagnosticados em 2002 e 2003 surtos e casos isolados em equinos de infecção por $T$. evansi em propriedades dos municípios de São Sepé e Alegrete [6,26,28]. Em 2007, mais um surto por T. evansi foi identificado em equinos no município de Cruz Alta [46]. Desse modo, este artigo teve o objetivo de realizar uma revisão sobre os surtos ocorridos de infecção por T. evansi em equinos no sul do Brasil.

\section{SURTOS DE TRIPANOSSOMOSE NO SUL DO BRASIL}

Neste estudo, compararam-se as descrições de artigos referentes à epidemiologia, sinais clínicos, exames laboratoriais, achados patológicos, diagnóstico e terapia de caso de infecção por T. evansi ocor- ridos em equinos no estado do Rio Grande do Sul. As diferenças entre os casos foram analisadas e discutidas entre si e com a literatura brasileira e internacional, dando ênfase para as novas descobertas referentes a este parasito em cavalos.

\section{NOVO CASO: Trypanosoma evansi EM EQUINO NO RS}

Em julho de 2009, no município de São Vicente do Sul, localizado na região central do estado do Rio Grande do Sul, foi avaliado um equino, fêmea com 11 anos de idade, que apresentava hipertermia, mucosas congestas e enrijecimento dos membros pélvicos. Este apresentava bom estado nutricional e apetite voraz. $\mathrm{O}$ animal era oriundo de uma propriedade que continha oito equinos, de sexo e idade variados, criados em pastagem nativa e utilizados para o trabalho de pecuária de corte.

A partir dos sinais clínicos, suspeitou-se de infecção por T. evansi. Foi realizado esfregaço sanguíneo periférico da extremidade distal da orelha de todos os equinos da propriedade, assim como coleta de sangue da veia jugular para análises hematológicas. O material foi processado hematologicamente, sendo avaliado o número de eritrócitos totais, hematócrito, hemoglobina, proteínas plasmáticas, fibrinogênio e leucograma completo. Foi também realizada a administração de $1 \mathrm{ml}$ de sangue do animal com enrijecimento dos membros pélvicos pela via intraperitonial em dois ratos Wistar. O sangue destes roedores foi coletado com anticoagulante e preservado em etanol (v/v) para extração do DNA e análise utilizando reação de polimerase em cadeia (PCR) para T. evansi específico [44].

O diagnóstico de tripanossomose foi determinado através da visualização de formas tripomastigotas de T. evansi nos esfregaços sanguíneos obtidos do equino doente. Os demais animais não apresentaram este flagelado em esfregaço sanguíneo e capa flogística de micro-hematócrito. A inoculação de sangue do equídeo em dois ratos confirmou a presença do protozoário flagelado. Este, isolado, foi iden- 
tificado como LPV-2009. Estes animais apresentaram parasitemia em média 11 dias após a inoculação. $\mathrm{O}$ isolado apresentou elevada patogenicidade, pois o sangue dos roedores demonstrou grande número de parasitos circulantes (Figura 1) e morte, em média, seis dias após o período pré-patente. O PCR realizado nos ratos confirmou a suspeita de T. evansi no equino.

Os parâmetros do eritrograma de todos os animais não estavam alterados, porém, somente no animal suspeito, foi observado um aumento na concentração de proteínas plasmáticas, fibrinogênio, no número de leucócitos totais devido ao aumento de neutrófilos segmentados e basófilos. Quando foi realizado o hematócrito, examinou-se a capa flogística para presença de hemoparasitos mas o T. evansi não foi visualizado.

Com a confirmação de tripanossomose optou-se pelo tratamento com aceturato de diminazeno. Porém, em consequência do animal estar no sexto mês de gestação, optou-se por uma dose única de $3,5 \mathrm{mg} \mathrm{kg}^{-1}$, como recomenda o fabricante, para evitar possível intoxicação e/ou aborto. Após tratamento com aceturato de diminazeno, o animal teve melhora dos sinais clínicos em até cinco dias após a terapia. Após 50 dias, o animal foi re-examinado, não sendo detectada qualquer alteração clínica. Os demais equídeos não foram medicados e não apresentaram sinais clínicos e o protozoário no esfregaço sanguíneo. Este pode ser um caso isolado de T. evansi, o que não é comum, conforme a literatura, porém esta afir-

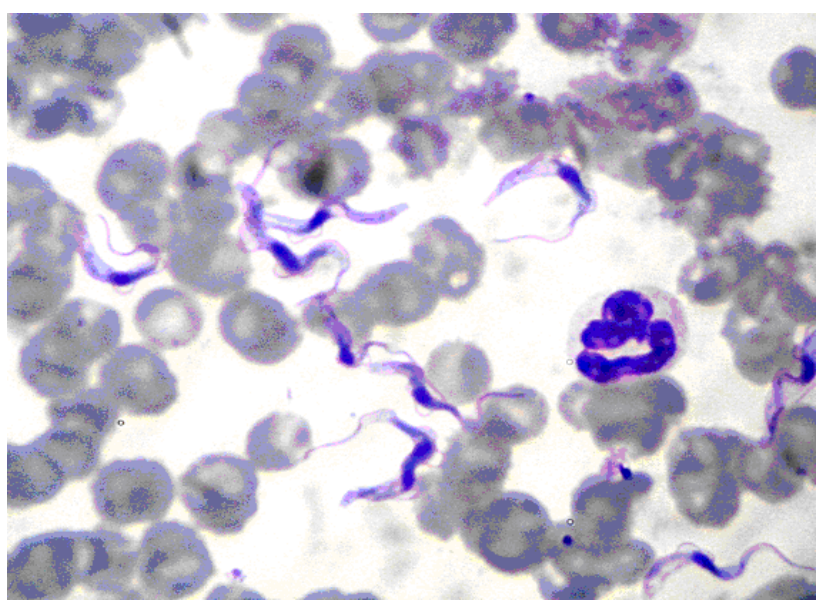

Figura 1. Rato infectado com T. evansi. Esfregaço sanguineo oriundo da extremidade distal da cauda de um rato após 17 dias da inoculação com sangue do cavalo com tripanossomose e corado com panótico rápido, (100x). $\mathrm{BAR}=25 \mu \mathrm{m}$. mação não pode ser feita, já que testes mais sensíveis como o PCR não foram realizados nos demais cavalos.

\section{DISCUSSÃO}

Este caso de T. evansi em equino no município de São Vicente do Sul, trata-se de um caso isolado, diferente do que ocorreu nos municípios de São Sepé, Alegrete [6,28] e Cruz Alta [46], onde a doença ocorreu em um número maior de animais em um mesmo período. Casos isolados similares a este estudo foram reportados em cães no estado do RS [5,15] e em um bovino no município de Videira, SC [7]. Entretanto, no caso de Santa Catarina, em bovinos os autores mencionaram a possibilidade de um surto, apesar do diagnóstico ter sido realizado em somente um animal. Esta suspeita foi baseada no relato de que 10 animais da propriedade adoeceram e apresentaram sintomatologia clínica compatível com tripanossomose, com morte, sem diagnóstico, de quatro bovinos [7] e melhora dos demais animais após terapia com aceturato de diminazeno. Recentemente, foi publicado o primeiro caso de Trypanosoma vivax na região sul do Brasil [11] em bovino, isoladamente, semelhante ao que ocorreu no equino em São Vicente do Sul.

Cães e bovinos podem atuar como reservatórios do T. evansi [20], porém, na propriedade de São Vicente do Sul, nenhuma destas espécies apresentou sinais clínicos compatíveis de tripanossomose. Nos surtos ocorridos em equinos no RS, a investigação epidemiológica apontou as capivaras como as fontes de infecção para os animais domésticos [6, $28,46]$, baseado na informação de que as capivaras e os quatis são considerados os principais reservatórios silvestres do T. evansi [20]. No caso ocorrido em São Vicente do Sul, as capivaras estavam presentes nas áreas de várzea da fazenda. Nos surtos em Alegrete e São Sepé $[6,26,28]$ também não se descartou a possibilidade da doença ter chegado à localidade pela circulação de equinos entre as propriedades, principalmente machos no período de monta. Nos casos de Cruz Alta [46] e de São Vicente do Sul, não havia registro de saída e entrada de equinos e bovinos, há mais de um ano, porém não se obtiveram informações sobre as propriedades vizinhas.

Os surtos de infecção por T. evansi em animais de produção no Brasil ocorrem de forma endêmica em regiões de clima quente [36]. No Rio 
Grande do Sul, a maioria dos casos de tripanossomose em equinos ocorreu no verão $[6,26,28,46]$, isso pode ser facilmente explicado pelo grande número de insetos hematófagos neste período, o que facilita a disseminação entre os animais do flagelado. $\mathrm{O}$ caso isolado ocorrido em São Vicente do Sul ocorreu no inverno e possivelmente outros animais não foram infectados devido ao número reduzidos de insetos vetores do parasito, em consequência do frio.

Nos relados ocorridos nos municípios de Alegrete e São Sepé, foram observados nos equinos sinais clínicos de emagrecimento progressivo (apesar de apetite voraz), letargia, incoordenação, instabilidade e atrofia dos membros pélvicos, dificuldade para levantar, fraqueza muscular, palidez das mucosas, edema subcutâneo e abortamento [6,26,28]. No surto em Alegrete, ainda identificou-se a sintomatologia nervosa de ataxia, andar em círculos, hiperexcitabilidade, cabeça inclinada, pressão contra a cabeça e movimentos de pedalagem [29]. O mesmo não ocorreu nos animais do município de Cruz Alta, que apresentavam ótimo estado nutricional [46], porém com enrijecimento dos membros pélvicos e dificuldade de locomoção, sintomas semelhantes ao do equino infectado de São Vicente do Sul. A literatura sugere a paralisia de membros pélvicos associada à caquexia como um quadro crônico da tripanossomose [33], porém isso não foi observado nos surtos de Cruz Alta e São Vicente do Sul. O mecanismo que leva à paralisia de membros pélvicos ainda não está elu-cidado, mas algumas novas pesquisas ligadas a alterações bioquímicas estão ocorrendo [12].

Em estudo recente realizado em gatos infectados com T. evansi foram observados sinais clínicos de incoordenação motora, claudicação e mortalidade associada à redução na atividade das enzimas acetilcolinesterase (AChE) e butirilcolinesterase (BuChE), no sistema nervoso central (SNC) e sistema vascular [12]. Conforme pesquisadores [39], a atividade da AChE é indispensável nas sinapses nervosas a fim de formar e modular o impulso, o que reflete na formação do movimento. Em cavalos, não há pesquisa da atividade destas enzimas nos animais infectados relacionadas com a sintomatologia clínica motora. Autores sugerem a encefalite necrosante em cavalos infectados como causa dos sinais clínicos neurológicos [29], alterações estas não encontradas nos gatos com T. evansi,
[12] que apresentaram sinais clínicos similares aos dos equinos.

A anemia é uma das alterações clínicas mais marcantes da infecção por T. evansi em cavalos [18, 25], porém sua patogênese não está completamente esclarecida. Estudos sugerem que a anemia não ocorre devido à lesão de medula óssea, mas sim pela destruição dos eritrócitos [16]. Outros autores consideram um mecanismo hemolítico imunomediado como o responsável pela anemia em infecções por T. evansi, associado com o desenvolvimento de complexos antígeno-anticorpo devido à aderência das proteínas de superfície do parasito nas células vermelhas $[1,2,25]$. Em estudos mais recentes, a anemia foi atribuída à enzima neuraminidase, que torna os glóbulos vermelhos propensos à fagocitose pelo sistema retí-culoendotelial [32]. O estresse oxidativo, demonstrado pela peroxidação lipídica, mostrou fragilidade na membrana do eritrócito pelo acúmulo de radicais livres [45]. Além de seu papel clássico na transmissão colinérgica, a enzima AChE tem demonstrado atividade hematopoiética pela sua presença em células progenitoras do sangue [31]. Assim, sugere-se que a inibição desta enzima, como verificado em gatos infectados com T. evansi [12], pode ser um dos mecanismos de ação relacionados com o processo anêmico dos casos de tripanossomose.

Contrariando a literatura, nos cavalos de Cruz Alta [46] e São Vicente do Sul, a anemia não foi observada, mas sim os sinais clínicos de hipertermia e dificuldade de locomoção. Nos demais casos brasileiros e do estado foi descrito redução de hematócrito $[6,24,26,28,33,34]$.

Alterações leucocitárias relatadas em equinos com tripanossomose por T. evansi incluem neutropenia [33], neutrofilia [24], monocitose [33,34], linfopenia [24] ou linfocitose [33,34]. Nos casos ocorridos no estado do RS, foi observado leucocitose em decorrência do aumento acentuado de linfócitos [28,46], monócitos [26] e eosinófilos [46]. Nos equinos de São Sepé, o número de leucócitos não estava alterado [6], porém foi observado presença de monócitos ativados, linfócitos reativos, eritrofagocitose e acentuado "rouleaux", que podem ser consequência de uma possível resposta frente à estimulação antigênica ocorrida pelo parasito. Pesquisadores, ao avaliarem cavalos infectados com T. evansi, encontraram em $27,8 \%$ dos animais linfócitos atí- 
picos, células com características morfológicas diferentes das consideradas normais para uma espécie, mas não neoplásicas [28]. Estas alterações não foram descritas em outros estudos de infecção por T. evansi, mas já foram evidenciadas em outras doenças por protozoários como toxoplasmose, babesiose e malária [3].

Nos casos ocorridos no RS, em somente duas situações realizou-se a necropsia de cavalos infectados com T. evansi. As lesões macroscópicas caracterizavam-se por icterícia generalizada da carcaça, aumento do tamanho dos linfonodos, espleno e hepatomegalia com hemorragias petequiais na superfície capsular e atrofia das grandes massas musculares dos membros pélvicos [26,28]. Histologicamente, em um caso isolado [26], foi descrito atrofia das capas cortical e medular dos linfonodos, com dilatação dos vasos linfáticos. Em um número maior de animais, ocorreu hiperplasia nos folículos linfóides, grande número de células plasmocitárias blásticas (plasmoblastos), eritrofagocitose e hemossiderose [28]. No fígado, havia infiltrado inflamatório mononuclear nos espaços porta, hiperplasia das células de Kupffer, necrose centrolobular moderada e hemossiderose. Nos equinos em que os músculos esqueléticos e os nervos periféricos foram examinados, havia infiltrado inflamatório mononuclear perineural com degeneração walleriana. Nos músculos da coxa ocorreu necrose flocular e hialina, alternada com regeneração de miofibras, infiltrado inflamatório mononuclear entre as fibras e hemossiderose acentuada. Estas alterações já eram conhecidas na infecção por T. evansi, entretanto, estudiosos [29] fizeram a descrição de lesões no SNC que anteriormente haviam sido sugeridas por Seiler et al. [30]. No presente trabalho, os autores observaram em nove equinos com sinais clínicos neurológicos, encefalite necrosante de maior gravidade na substância branca associada a edema, desmielinização e infiltrado linfoplasmocitário perivascular. Estes dois únicos casos de lesão encefálica em cavalos com tripanossomose juntam-se aos ocorridos em bovinos e cervos infectados por $T$. evansi $[40,41]$.

O diagnóstico da tripanossomose em equinos no RS foi comprovado pela morfologia e biometria das formas tripomastigotas em esfregaço sanguíneo corado com Giemsa ou Panótico rápido [6,26,28,46]. Alguns autores fizeram o uso de outras ferramentas para confirmação do diagnóstico, como sorologia e imuno histoquímica [28], xenodiagnós-tico [46] e PCR específico para T. evansi, no caso isolado de São Vicente. Na técnica de imuno his-toquímica, foram visualizados tripomastigotas no encéfalo de animais com alterações neurológicas. O isolamento e replicação de tripomastigotas de $T$. evansi em ratos da linhagem Wistar foi um método confiável de diagnóstico nos equinos de Cruz Alta e São Vicente do Sul. O PCR é uma das técnicas mais sensíveis para detectar e confirmar infecção por Trypanossoma em animais, pois além da especi-ficidade, permite a diferenciação entre espécies. Este método foi indispensável para confirmação do primeiro caso de T. vivax no RS [11].

No surto ocorrido em Alegrete, mais de 100 equinos adoeceram e 60 morreram em decorrência da tripanossomose [28]. Porém, muitos destes animais foram medicados com drogas tripanocidas como o aceturato de diminazeno, cloridrato de diamidina e diclazuril nas doses recomendadas pelos fabricantes. Em outro caso, o cavalo medicado com cloridrato de diamidina [26] apresentou novamente os sinais clínicos associados à recidiva da parasitemia. No Pantanal matogrossense onde a tripanossomose é endêmica, os prejuízos anuais com morte de animais são grandes, apesar do tratamento. Muitos fazendeiros fazem o uso de terapia profilática à base de aceturato de diminazeno e assim conseguem reduzir o número de equinos doentes $[33,34,36]$. Em um grande surto na Tailândia, os animais de uma fazenda foram medicados com uma associação de fármacos (ace-turato de diminazeno + suramim) que não teve eficácia curativa, resultando na mortalidade de um grande número de animais e abortamento de $40 \%$ das éguas [42].

A ineficácia do aceturato de diminazeno é atribuída ao tamanho das moléculas do fármaco, que não são capazes de atravessar a barreira hematoencefálica, onde o flagelado fica abrigado ou ultrapassa em uma quantidade insuficiente para combater o protozoário [17,37]. Portanto, o diminazeno controla o flagelado na circulação por um período de 21 dias, em média (período residual da droga), mas não no SNC [22]. Baseado na possibilidade de que um pequeno número de moléculas de aceturato de diminazeno ultrapassa a barreira hematoencefálica, pesquisadores testaram um novo protocolo 
terapêutico com três doses de $7 \mathrm{mg} \mathrm{kg}^{-1}$ em intervalo de 7 dias e obtiveram a cura clínica de todos os animais doentes [10]. Testes semelhantes foram realizados em outros animais infectados experimentalmente por $T$. evansi (cinco doses de 3,5 $\mathrm{mg} \mathrm{kg}^{-1}$ em intervalos de $24 \mathrm{~h}$ ), onde o porcentual de cura foi de $100 \%$ para ratos e $87,5 \%$ para gatos $[9,13]$.

Apesar da infecção por T. evansi ter sido diagnosticada pela primeira vez no RS no ano de 2002, veterinários de campo têm reportado achados clínicos como paralisia de membros pélvicos, febre e emagrecimento desde os anos 80 e 90 (Comunicação Pessoal), mas provavelmente o desconhecimento do T. evansi retardou seu primeiro registro. Outra hipótese seria a suspeita de babesiose nos animais, pois ambas as enfermidades têm a anemia e hipertermia como características marcantes, e como o aceturato de diminazeno tem ação tripanocida e babesicida, possivelmente os equinos curaram-se da tripanossomose, mesmo tendo diagnóstico clínico de babesiose.

\section{CONSIDERAÇÕES FINAIS}

Com base na literatura, observamos que o diagnóstico de tripanossomose tem sido mais frequente, sendo possivelmente os animais silvestres a fonte de infecção para os animais domésticos. Entre os surtos, foi verificado que os sinais clínicos e os achados de patologia clínica são relacionados principalmente ao sistema locomotor e à anemia. Achados patológicos importantes e raramente reportados em equinos foram realizados no surto ocorrido no município de Alegrete, como lesões no SNC associado aos sinais clínicos neurológicos. Outra informação pertinente foi o novo protocolo terapêutico com sucesso terapêutico de $100 \%$ no caso do município de Cruz Alta. As técnicas de diagnóstico utilizadas nos animais de produção foram eficientes, isto é, apresentaram grande especificidade, porém têm baixa sensibilidade. A região Sul carece de pesquisas relacionadas à tripanossomose. Estudos de prevalência, com técnicas mais sensíveis, são necessários, com a finalidade de determinar a abrangência desta doença e os prejuízos econômicos causados aos pecuaristas.

\section{REFERÊNCIAS}

1 Assoku R.K.G. 1975. Immunological studies of the mechanism of anaemia in experimental Trypanosoma evansi infection in rats. International Journal Parasitology. 5(2): 137-145.

2Audu P.A., Esievo K.A., Mohamed G. \& Ajanusi O.J. 1999. Studies of infectivity and pathogenicity of an isolate of Trypanosoma evansi in Yankasa sheep. Veterinary Parasitology. 86(3): 185-190.

3 Bain B.J. 1997. Células Sangüineas: Um Guia Prático. Porto Alegre: ArtMed, 334p.

4 Brender G.C., Pugh D.M., Bywater R.J. \& Jenkins W.L. 1991. Veterinary Applied Farmacology \& therapeutics. Toronto: Balliere Tindall/Saunders, 624p.

5 Colpo C.B., Colpo E.T.B., Stainki D.R. \& Monteiro S.G. 2005. Infecção Natural por Trypanosoma evansi em cão no Rio Grande do Sul. Ciência Rural. 35(3): 717-719.

6 Conrado A.C., Lopes S.T.A., Oliveira L.S.S., Monteiro S.G., Batista D.L. \& Bueno A. 2005. Infecção natural por Trypanosoma evansi em cavalos na região central do estado do Rio Grande do Sul. Ciência Rural. 35(4): 928-931.

7 Da Silva A.S., Oliveira C.B., Zanette R.A., Soares C.D.M., Coradini G.P., Polenez C.H., Santurio J.M. \& Monteiro S.G. 2007. Ocorrência de Trypanosoma evansi em bovinos de uma propriedade leiteira no município de Videira SC. Acta Scientiae Veterinariae. 35(3): 373-376.

8 Da Silva A.S., Zanette R.A., Colpo C.B., Santurio J.M. \& Monteiro S.G. 2008. Sinais Clínicos em cães naturalmente infectados por Trypanosoma evansi (Kinetoplastida: Trypanosomatidae) no Rio Grande do Sul, Brasil. Clínica Veterinária. 8(72): 6668.

9 Da Silva A.S., Tochetto C., Zanette R.A., Pierezan F., Rissi D.R., Santurio J.M. \& Monteiro S.G. 2008. Aceturato de diminazeno e dipropionato de imidocarb no controle de infecção por Trypanosoma evansi em Rattus norvegicus infectados experimentalmente. Ciência Rural. 38(5): 1357-1362.

10 Da Silva A.S., Zanette R.A., Otto M.A., Pereira P.L., Gressler L.T. \& Monteiro S.G. 2009. Aceturato de diminazeno no tratamento de equinos infectados naturalmente por Trypanosoma evansi no município de Cruz Alta - RS, Brasil. Veterinária e Zootecnia. 16(1): 74-79.

11 Da Silva A.S., Costa M.M., Polenz M.P., Polenz C.H., Teixeira M.M.G., Lopes S.T.A. \& Monteiro S.G. 2009. Primeiro registro de Trypanosoma vivax em bovinos no estado do Rio Grande do Sul, Brasil. Ciência Rural. 38(8): 2550-2554. 
Silva A.S., Andrade Neto O.A.S., Costa M.M., Wolkmer P., Mazzantti C.M., Santurio J.M., Lopes S.T.A. \& Monteiro S.G. 2010.

12 Da Silva A.S., Spanevello R., Stefanello N., Wolkmer P., Costa M.M., Zanette R.A., Lopes S.T.A., Santurio J.M., Schetinger M.R.C. \& Monteiro S.G. 2009. Influence of Trypanosoma evansi in blood, plasma, and brain cholinesterase of experimentally infected cats. Research Veterinary Science. In press.

13 Da Silva A.S., Zanette R.A., Wolkmer P., Costa M.M., Teixeira M.M.G., Santurio J.M., Lopes S.T.A. \& Monteiro S.G. 2009. Diminazene aceturate in the control of Trypanosoma evansi infection in cats. Veterinary Parasitology. 165(1-2): 47-50.

14 DávilaA.M.R. \& Silva R.A.M.S. 2000. Animal trypanosomiasis in South America. Current status, partnership, and information technology. Annals of the New York Academy of Sciences. 916(1): 199-212.

15 Franciscato C., Lopes S.T.A., Teixeira M.M.G., Wolkmer P., Paim B.C. \& Monteiro S.G. 2007. Cão naturalmente infectado por Trypanosoma evansi em Santa Maria, RS, Brasil. Ciência Rural. 37(1): 288-291.

16 Jatkar P.R. \& Purohit M.S. 1971. Pathogenesis of anaemia in T. evansi infection. I. Haematology. Indian Veterinary Journal. 48(3): 239-244.

17 Jenings F.W., Whitelaw D.D. \& Urquhart G.M. 1977. The relationship between duration of infection with T. brucei in mice and the efficacy of chemotherapy. Parasitology. 75(2): 145-153.

18 Jenkins G.C. \& Facer C.A. 1985. Hematology of African trypanosomiasis. In: Tizard I. (Ed.) Immunology and Pathogenesis of Trypanosomiasis. Boca Raton: CRC Press, p.13-44.

19 Joshi P.P., Shegokar V.R., Powar R.M., Herder S., Katti R., Salkar H.R., Dani V.S., BhargavaA., Jannin J. \& Truc. P. 2005. Human trypanosomosis caused by Trypanosoma evansi in India: The first case report. American Journal of Tropical Medicine and Hygiene. 73(3): 491-495.

20 Herrera H.M., Dávila A.M.R., Norek A., Abreu U.G., Souza S.S., D’Andrea P.S. \&. Jansen A.M. 2004. Enzootiology of Trypanosoma evansi in Pantanal, Brazil. Veterinary Parasitology. 125(3-4): 263-275.

21 Hoare C.A. 1972. The Trypanosomes of mammals: a zoological monograph. Blackwell: Oxford, 749p.

22 Kaminsky R. \& Brun R. 1998. In vivo and In vitro activities of trybizine hydrochloride against various pathogenic trypanosome species. Antimicrobial Agents and Chemotherapy. 42(11): 2858-2863.

23 Losos G.J. 1980. Diseases caused by Trypanosoma evansi, a review. Veterinary Research Communications. 4(1): 165-181.

24 Marques L.C., Machado R.Z., Alessi A.C., Aquino L.P.C.T. \& Pereira G.T. 2000. Experimental infection with Trypanosoma evansi in horses: clinical and haematological observations. Revista Brasileira de Parasitologia Veterinária. 9(1): 11-15.

25 Maudlin I., Holmes P.H. \& Miles M.A. 2004. The Trypanosomiases. London: CABI pubhishing, 648p.

26 Moraes C.M., Curcio B.R., Ribas L.M., Nizoli L.Q. \& Nogueira C.E.W. 2007. Infecção por Trypanosoma evansi em equinos do Brasil. Revista Portuguesa de Ciência Veterinária. 102(1): 159-163.

27 Peregrine A.S. \& Mamman M. 1993. Pharmacology of Dimmenazene: a review. Acta Tropica. 54(1): $185-203$.

28 Rodrigues A., Fighera R.A., Souza T.M., SchildA.L., Soares M.P., Milano J. \& Barros C.S.L. 2005. Surtos de tripanossomíase por Trypanosoma evansi em equinos no Rio Grande do Sul: aspectos epidemiológicos, clínicos, hematológicos e patológicos. Pesquisa Veterinária Brasileira. 25(4): 239-249.

29 Rodrigues A., Fighera R.A., Souza T.M., Schild A.L. \& Barros C.S.L. 2009. Neuropathology of naturally occurring Trypanosoma evansi infection of horses. Veterinary Pathology. 46(2): 251-258.

30 Seiler R.J., Omar S. \& Jackson A.R. 1981. Meningoencephalitis in naturally occurring Trypanosoma evansi infection (surra) of horses. Veterinary Pathology. 18(1): 120-122.

31 Serobyan N., Jagannathan S., Orlovskaya I., Schraufstatter I., Skok M., Loring J. \& Khaldoyanidi S. 2007. The cholinergic system is involved in regulation of the development of the hematopoietic system. Life Science. 80(24-25): 2352-2360.

32 Shehu S.A., Ibrahim N.D.G., Esievo K.A.N. \& Mohammed G. 2006. Neuraminidase (Sialidase) activity and its role in development of anaemia in Trypanosoma evansi infection. Journal Applied Science. 6(13): 2779-2783.

33 Silva R.A.M.S., Arosemena N.A.E., Herrera H.M., Sahib C.A. \& Ferreira M.S. 1995. Outbreak of trypanosomosis due to Trypanosoma evansi in horses of Pantanal Mato-grossense, Brazil. Veterinary Parasitology. 60(1-2): 167-171.

34 Silva R.A.M.S., Herrera H.M., Domingos L.B.S., Ximenes F.A. \& Dávila A.M.R. 1995. Pathogenesis of Trypanosoma evansi infection in dogs and horses: hematological and clinical aspects. Ciência Rural. 25(2): 233-238.

35 Silva R.A.M.S., Barros A.T.M. \& Herrera H.M. 1995. Trypanosomosis outbreaks due to Trypanosoma evansi in the Pantanal: a preliminary approach on risk factors. Revue d'Élevage et de Médecine Vétérinaire des Pays Tropicaux. 48(4):315-319.

36 Silva R.A.M.S., Seidl A., Ramirez L. \& Dávila A.M.R. 2002. Trypanosoma evansi e Trypanosoma vivax: biologia diagnóstico e controle. Capturada em 15 jan. 2005. Disponível na Internet http://www.cpap.embrapa.br/publicacoes/online/ Livro015. 
37 Spinosa H.S., Górniak S.L. \& Bernardi M.M. 1999. Farmacologia aplicada à medicina veterinária. Rio de Janeiro: Guanabara koogan, 946p.

38 Taylor K. \& Authié E.M.L. 2004. Pathogenesis of animal trypanosomiasis. In: Maudlin I., Holmes P.H. \& Miles M.A. (Eds). The Trypanosomiases. London: CABI pubhishing, pp.331-354.

39 Thiermann H., Szinicz L., Eyer P., Zilker T. \& Worek F. 2005. Correlation between red blood cell acetylcholinesterase activity and neuromuscular transmission in organophosphate poisoning. Chemical biology Interactions. $158(2)$ : 345-347.

40 Tuntasuvan D., Sarataphan N. \& Nishikawa H. 1997. Cerebral trypanosomiasis in native cattle. Veterinary Parasitology. 73(3): 357-363.

41 Tuntasuvan D., Mimapan S., Sarataphan N., Trongwongsa L., Intraraksa R. \& Luckins A.G. 2000. Detection of Trypanosoma evansi in brains of the naturally infected hog deer by immunohistochemistry. Veterinary Parasitology. 87(2-3): 223-230.

42 Tuntasuvan D., Jarabrum W., Viseshakul N., Mohkaew K., Borisutsuwan S., Theeraphan A. \& Kongkanjana N. 2003. Chemotherapy of surra in horses and mules with diminazene aceturate. Veterinary Parasitology. 110(3): $227-233$.

43 Ventura R.M., Takata C.S., Silva R.A., Nunes V.L., Takeda G.F. \& Teixeira M.M. 2000. Molecular and morphological studiesof brazilian Trypanosoma evansi stocks: The toal absence of kDNA in trypanosomes from both laboratory stocks and naturally infected domestic and wild mammals. Journal of Parasitology. 86(6): 1289-1298.

44 Ventura R.M., Takeda G.F., Silva R.A.M.S., Nunes V.L.B., Buck G.A. \& Teixeira M.M.G. 2002. Genetic relatedness among Trypanosoma evansi stocks by random amplification of polymorphic DNA and evaluation of a synapomorphic DNA fragment for species-specific diagnosis. International Journal of Parasitology. 32(1): 53-63.

45 Wolkmer P., Da Silva A.S., Carnelutti J.F., Paim F.C., Traesel C., Lopes S.T.A. \& Monteiro S.G. 2009. Lipid peroxidation associated with anemia in rats experimentally infected with Trypanosoma evansi. Veterinary Parasitology. 165(1-2): 41-46.

46 Zanette R.A., Da Silva A.S., Costa M.M., Monteiro S.G., Santurio J.M. \& Lopes S.T.A. 2008. Ocorrência de Trypanosoma evansi em eqüinos no município de Cruz Alta, RS, Brasil. Ciência Rural. 38(5): 1468-1471. 